\title{
Effects of antioxidant supplement and metformin therapy on C-reactive protein and oxidative stress in patients with type 2 diabetes mellitus
}

\author{
Zeina A Munim Al-Thanoon \\ Department of Pharmacology and Toxicology, College of Pharmacy, University of Mosul, Mosul, Iraq.
}

\begin{abstract}
$\underline{\text { Received }}$
$\underline{\text { Accepted }}$

12.1.2013

28.4.2013
\end{abstract}

\begin{abstract}
Objectives: The study aims to compare the effects of metformin and antioxidant vitamins $\mathrm{C}$ and $\mathrm{E}$ on C-reactive protein (CRP) and oxidative/antioxidant status (represented by serum malondialehyde (MDA) and total antioxidant status (TAS) in newly diagnosed patients with Type 2 diabetes mellitus (T2DM) at baseline and after 3 months of therapy compared with the control group.
\end{abstract}

Patients and Methods: The study was performed in Al-Wafaa Center of Diabetes Management and Research in Mosul, Iraq. They included 91 type 2 diabetic patients and 50 healthy controls. The patients were divided into two groups: The first group $(n=44)$ was received metformin alone orally for duration of 3 months and the second group ( $\mathrm{n}=$ 47) was received metformin with vitamin $C$ and $E$ orally for duration of 3 months also. Fasting blood samples were obtained from both controls and patients (before and after treatment) and assays were done for determination of serum MDA, TAS, and CRP.

Results: Compared with controls, patients serum levels of MDA, and CRP were significantly high and TAS were significantly lower. After 3 months of treatment, both patients groups reported significant reductions in serum levels of MDA, CRP and a significant increase in serum levels of TAS, compared with the pre-treatment levels. The present study found that there was a non significant difference in serum MDA levels between the two patients groups $(\mathrm{p}=0.026)$, whereas serum TAS levels was significantly higher and CRP was significantly lower in the second group as compared to the first group.

Conclusion: The present study concluded that three months oral consumption of combined antioxidant vitamins $\mathrm{C}$ and $\mathrm{E}$ with metformin in patients with T2DM showed significantly beneficial effect on oxidative stress and inflammatory response compared with metformin alone. The findings of this study necessitate investigating the overall clinical impact of antioxidant treatment of type 2 diabetes mellitus to maintain good glycemic control.

Keywords: Type 2 Diabetes mellitus; Antioxidant vitamins (E and C); Metformin; oxidative/antioxidant status; C-reactive protein .

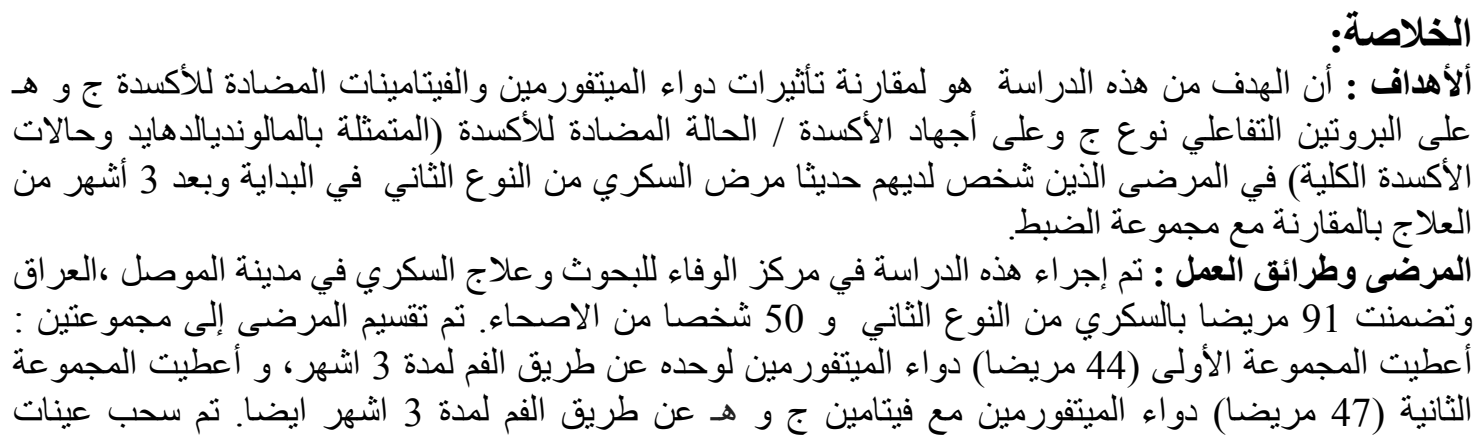




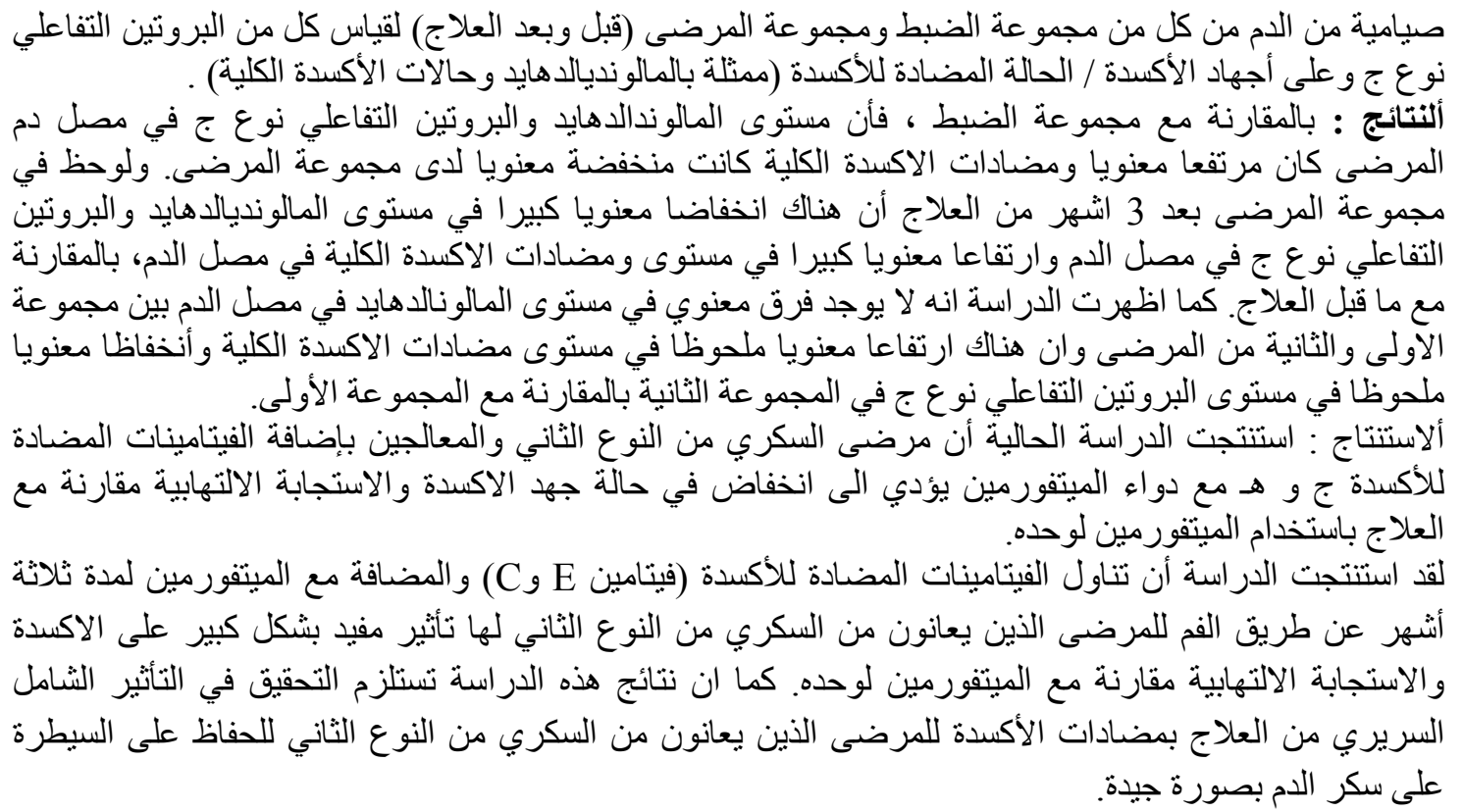

$\mathrm{T}$ he inter-relationships between glycemic control and progression of diabetes-related vascular complications, oxidative/antioxidative status and inflammation, have not been fully understood ${ }^{1,2,3}$ It has been known that the major factor which are responsible for the enhanced free radical generation in diabetic patients is hyperglycemia through autooxidation of glucose and production of advanced glycation end products (AGEs), which is known to progress at an extremely accelerated rate in T2DM. ${ }^{4,5,6}$ A proinflammatory state, recognized clinically by elevations of C-reactive protein (CRP) which is commonly present in patients with T2DM. Multiple mechanisms seemingly underlie elevations of CRP; one cause is obesity, because excess adipose tissue releases inflammatory cytokines that may elicit higher CRP levels. No drugs that act exclusively via this mechanism are available for reducing cardiovascular risk. However, several drugs are used to treat other metabolic risk factors in
T2DM have been reported to reduce CRP levels. These drugs groups are statins, nicotinic acid, fibrates and thiazolidinediones. $^{7,8}$

Drugs most commonly used for T2DM are sulfonylureas, metformin, and insulin-sensitizing glitazones, in which all have been associated with normalization of circulating inflammation and oxidative markers. This effect may be mediated, at least partly, by the action of the therapeutic agents on the innate immune system to retain the homeostasis of the oxidative and inflammatory status. ${ }^{9}$ Metformin is recommended as an oral hypoglycemic core therapy in diabetes management worldwide and the guidelines of the American Diabetes Association (ADA) and the European Association for the Study of Diabetes (EASD) recommend its use in patients irrespective of age, body weight and degree of baseline hyperglycemia, due to its favorable effect on metabolic indices of glucose, lipid, and weight control. ${ }^{10,11}$ 
Many micronutrients exhibit well-characterized anti-inflammatory or immunomodulatory functions. Vitamin C (ascorbic acid) is the primary hydrophilic antioxidant found in human plasma. ${ }^{12}$ It has an important role in immune function and various oxidative/inflammatory processes, such as scavenging reactive oxygen species and reactive nitrogen species, preventing the initiation of chain reactions that lead to protein glycation, and protecting against lipid peroxidation. ${ }^{13,14,15}$ Vitamin $\mathrm{E}$, a component of the total peroxyl radical-trapping antioxidant system, reacts directly with peroxyl and superoxide radicals and singlet oxygen and protects membranes from lipid peroxidation. ${ }^{16,17,18}$

The aim of this study was to compare and determine the effects of metformin combination with antioxidant vitamins $\mathrm{C}$ and $\mathrm{E}$ on acute phase protein (represented by serum C-reactive protein(CRP), oxidative/antioxidant status (represented by serum malondialehyde (MDA) and total antioxidant status (TAS) in newly diagnosed patients with T2DM at baseline and after 3 months of therapy.

\section{Patients And Methods}

The study protocol was approved by regional research ethics committees at College of Pharmacy and Mosul Health Administration. It is a follow up study, performed during the period between $1 / 6 / 2012$ and $1 / 10 / 2012$.

Patients included in this study were collected from Al-Wafaa Center of Diabetes Management and Research in Mosul, Iraq. Inclusion criteria include ninety one newly-diagnosed patients with T2DM , their ages ranged between 30 and 65 years old including both males and females. Their selection was according to the American Diabetes Association (ADA) ${ }^{19}$ and the World Health Organization (WHO) ${ }^{20}$ diagnostic criteria for T2DM. They were divided into two groups: The first group $(\mathrm{n}=$ 44) was received metformin orally alone (Dialon, Julphar, United Arab Emirates), $500 \mathrm{mg}$ three times daily alone for duration of 3 months. They were 24 females and 20 males with a mean age of $53.15 \pm 5.01$ years, and the second group $(\mathrm{n}=47)$ was received metformin $500 \mathrm{mg}$ orally three times daily in combination with vitamin supplements $(250 \mathrm{mg}$ vitamin $\mathrm{C}$ three times daily and $400 \mathrm{IU}$ vitamin $\mathrm{E}$ daily) for duration of 3 months. They were 26 females and 21 males with a mean age of $50.35 \pm 4.52$ years. Exclusion criteria was patients with history of acute and chronic inflammatory illness, cardiovascular disease, renal failure and age range $30<$ age $>65$ years old . Females included in the study were neither being pregnant nor lactating, nor menstruating at the time of blood collection.

Fifty apparently healthy volunteers (26 females and 24 males, with a mean age of $42.56 \pm 5.64$ years, and with no previous history of diabetes mellitus, were included as a control group to establish the normal values for MDA, TAS and CRP.

Venous blood samples were drawn under fasting condition from diabetic patients before and after three month of treatment. Blood samples from the healthy control subjects were collected and processed in the same way.

Serum MDA levels were estimated using a thiobarbituric acid (TBA) assay, ${ }^{21}$ and TAS according to the method described by Miller et $a l .,{ }^{22}$ using a Randox TAS kit (Randox Laboratories Ltd., Antrim, UK). Serum $\mathrm{C}$-reactive protein was measured by 
enzyme-linked immunosorbent assay (ELISA), using BioCheck CRP ELISA kit (BioCheck Inc., California, USA).

Statistical analysis of the data, were expressed as mean \pm standard deviation (SD). Unpaired t-test is used to compare between baseline data of the control group and other groups. Paired t-test is used to compare between data of before treatment and after treatment of metformin group and combination group. The statistical results were considered significant at $p \leq 0.05 .^{23}$

\section{Results}

The serum levels of MDA and CRP were significantly higher $(\mathrm{p}<0.001)$ and the serum level of TAS was significantly lower $(p<0.001)$ in patients with T2DM before starting therapy with metformin alone or in combination with vitamins $\mathrm{C}$ and $\mathrm{E}$ (at baseline) in comparison with the control group (Table 1 and 3 respectively). After three months of therapy with metformin alone, the serum levels of MDA and CRP although reduced but still there were significant differences from the control values (Table 1 and 3 respectively). After three months of therapy with metformin alone, the serum levels of MDA and CRP were reduced and the serum level of TAS were increased but still there was a significant differences $(p<0.01)$ from the control values (Table 1 and 3 respectively). Regarding patients treated with metformin in combination with vitamin $\mathrm{E}$ and $\mathrm{C}$, there were highly significant reduction $(p<0.001)$ in the serum levels of MDA and CRP and a highly significant increase $(p<0.001)$ in the serum level of TAS as compared to the control values (Table 1 and 3 respectively).

Comparison of serum levels of MDA and CRP before and after therapy with metformin alone, show significant reductions $(\mathrm{p}<0.01)$ in MDA and CRP and a significant increase $(p<0.01)$ in TAS after three months of therapy (Table 2). While comparison of serum levels of TAS in patients with T2DM before and after therapy with metformin in combination with vitamin $\mathrm{E}$ and $\mathrm{C}$, show highly significant reductions $(\mathrm{p}<0.001)$ in MDA and CRP and a highly significant increase $(p<0.001)$ in TAS after three months of therapy had been obtained (Table 4).

Table 1: Comparison of MDA, TAS and CRP between control and patient groups (before and after metformin therapy).

\begin{tabular}{|c|c|c|c|}
\hline \multirow{2}{*}{ Parameter } & \multicolumn{3}{|c|}{ Mean \pm SD } \\
\cline { 2 - 4 } & $\begin{array}{c}\text { Control } \\
(\mathbf{n}=\mathbf{5 0})\end{array}$ & $\begin{array}{c}\text { Before Drug } \\
(\mathbf{n}=\mathbf{4 4})\end{array}$ & $\begin{array}{c}\text { After Drug } \\
(\mathbf{n}=\mathbf{4 4})\end{array}$ \\
\hline MDA $(\mu \mathrm{mol} / \mathrm{L})$ & $1.05 \pm 0.13$ & $2.10 \pm 0.24^{* * *}$ & $1.90 \pm 0.20^{* *}$ \\
\hline TAS $(\mathrm{mmol} / \mathrm{L})$ & $1.91 \pm 0.11$ & $1.06 \pm 0.15^{* * *}$ & $1.14 \pm 0.13^{* *}$ \\
\hline CRP $(\mathrm{mg} / \mathrm{L})$ & $0.48 \pm 0.07$ & $1.66 \pm 0.12^{* * *}$ & $1.52 \pm 0.10^{* *}$ \\
\hline
\end{tabular}

$* * *$ Significant difference from control at $\mathrm{p} \leq 0.001, * *$ Significant difference from control at $\mathrm{p} \leq 0.01$ using unpaired t- test. 
Table 2: Effects of metformin therapy on MDA, TAS and CRP in patients group with T2DM.

\begin{tabular}{|c|c|c|c|}
\hline \multirow{2}{*}{ Parameter } & \multicolumn{3}{|c|}{ Mean \pm SD } \\
\cline { 2 - 4 } & Before & After & p-value \\
\hline MDA $(\mu \mathrm{mol} / \mathrm{L})$ & $2.10 \pm 0.24$ & $1.90 \pm 0.20$ & $<0.01$ \\
\hline TAS $(\mathrm{mmol} / \mathrm{L})$ & $1.06 \pm 0.15$ & $1.14 \pm 0.13$ & $<0.01$ \\
\hline CRP $(\mathrm{mg} / \mathrm{L})$ & $1.66 \pm 0.12$ & $1.52 \pm 0.10$ & $<0.01$ \\
\hline
\end{tabular}

Using paired t- test $(n=44)$.

Table 3:Comparison of MDA, TAS and CRP between control and patients group (before and after metformin-vitamin combination therapy).

\begin{tabular}{|c|c|c|c|}
\hline \multirow{2}{*}{ Parameter } & \multicolumn{3}{|c|}{ Mean \pm SD } \\
\cline { 2 - 4 } & $\begin{array}{c}\text { Control } \\
\mathbf{( n = 5 0 )}\end{array}$ & $\begin{array}{c}\text { Before Drug } \\
(\mathbf{n}=\mathbf{4 7})\end{array}$ & $\begin{array}{c}\text { After Drug } \\
\mathbf{( n = 4 7 )}\end{array}$ \\
\hline MDA $(\mu \mathrm{mol} / \mathrm{L})$ & $1.05 \pm 0.13$ & $2.08 \pm 0.25^{* * *}$ & $1.83 \pm 0.19^{* * *}$ \\
\hline TAS $(\mathrm{mmol} / \mathrm{L})$ & $1.91 \pm 0.11$ & $1.08 \pm 0.17^{* * *}$ & $1.29 \pm 0.14^{* * *}$ \\
\hline CRP $(\mathrm{mg} / \mathrm{L})$ & $0.48 \pm 0.07$ & $1.54 \pm 0.11^{* * *}$ & $1.28 \pm 0.26^{* * *}$ \\
\hline
\end{tabular}

$* * *$ Significant difference from control at $\mathrm{p} \leq 0.001$ using unpaired $\mathrm{t}-$ test.

Table 4: Effects of metformin-vitamin combination on MDA, TAS and CRP in patients group with T2DM.

\begin{tabular}{|c|c|c|c|}
\hline \multirow{2}{*}{ Parameter } & \multicolumn{3}{|c|}{ Mean \pm SD } \\
\cline { 2 - 4 } & Before & After & p-value \\
\hline MDA $(\mu \mathrm{mol} / \mathrm{L})$ & $2.08 \pm 0.25$ & $1.83 \pm 0.19$ & $<0.001$ \\
\hline TAS $(\mathrm{mmol} / \mathrm{L})$ & $1.08 \pm 0.17$ & $1.29 \pm 0.14$ & $<0.001$ \\
\hline $\mathrm{CRP}(\mathrm{mg} / \mathrm{L})$ & $1.54 \pm 0.11$ & $1.28 \pm 0.26$ & $<0.001$ \\
\hline
\end{tabular}

Using paired t- test $(n=47)$.

Table 5: Comparison of percent of variation of the studied parameters after therapy with metformin alone or in combination with vitamins $(\mathrm{E}+\mathrm{C})$.

\begin{tabular}{|l|c|c|c|}
\hline \multirow{2}{*}{ Parameter } & \multicolumn{2}{|c|}{ Mean \pm SD (\%) } & \multirow{2}{*}{ p-value } \\
\cline { 2 - 3 } & $\begin{array}{c}\text { Metformin } \\
(\mathbf{n}=\mathbf{4 1})\end{array}$ & $\begin{array}{c}\text { Metformin+ Vitamins } \\
(\mathbf{n}=\mathbf{4 7})\end{array}$ & \\
\hline MDA $(\mu \mathrm{mol} / \mathrm{L})$ & $-0.20 \pm 0.04$ & $-0.25 \pm 0.06$ & $(\mathrm{NS})$ \\
\hline TAS $(\mathrm{mmol} / \mathrm{L})$ & $0.08 \pm 0.02$ & $0.21 \pm 0.15$ & $<0.001$ \\
\hline CRP $(\mathrm{mg} / \mathrm{L})$ & $-0.14 \pm 0.02$ & $-0.26 \pm 0.15$ & $<0.01$ \\
\hline
\end{tabular}

$\mathrm{NS}=$ Not significant using unpaired t-test; Negative sign means decrease. 
Table 6: Correlations between MDA and TAS in patients with T2DM.

\begin{tabular}{|c|c|c|}
\hline \multirow{2}{*}{ Parameter } & \multicolumn{2}{|c|}{ TAS $(\mathrm{mmol} / \mathrm{L})$} \\
\cline { 2 - 3 } & $\mathbf{r}$ & p-value \\
\hline MDA $(\mu \mathrm{mol} / \mathrm{L})$ & -0.327 & $<0.001$ \\
\hline
\end{tabular}

Table 7: Correlations between MDA, TAS and CRP in patients with T2DM.

\begin{tabular}{|c|c|c|c|c|}
\hline \multirow{2}{*}{ Parameter } & \multicolumn{2}{|c|}{$\mathrm{MDA}(\mu \mathrm{mol} / \mathrm{L})$} & \multicolumn{2}{c|}{$\mathrm{TAS}(\mathrm{mmol} / \mathrm{L})$} \\
\cline { 2 - 5 } & $\mathbf{r}$ & $\mathbf{p}-$ value & $\mathbf{r}$ & $\mathbf{p}$-value \\
\hline $\mathrm{CRP}(\mathrm{mg} / \mathrm{L})$ & 0.892 & $<0.001$ & -0.731 & $<0.001$ \\
\hline
\end{tabular}

Table (5) shows a comparison between the percent of variation of the studied parameters between the two patients groups. A non significant difference had been obtained with the serum levels of MDA $(p=0.026)$ and there are significant differences in the serum levels of TAS $(p<0.001)$ and CRP $(p<0.01)$ between the two patients groups.

Regarding correlations, there was a highly significant negative correlation between TAS and MDA levels ( $\mathrm{r}=$ $0.327 ; p<0.001$ ) (Table 6), and between TAS and CRP levels ( $\mathrm{r}=-0.731$; $\mathrm{p}<0.001$ ) in T2DM patients (Table 7), while there was a highly significant positive correlation between CRP and MDA levels $(\mathrm{r}=0.892 ; \mathrm{p}<0.001)$ in T2DM patients (Table 7).

\section{Discussion}

The present study demonstrated significantly high serum MDA level and a significantly low serum TAS level in newly diagnosed patients with T2DM as compared to healthy controls. It was also demonstrated that the administration of metformin alone or in combination with vitamins $\mathrm{C}$ and $\mathrm{E}$ to such patients for a duration of 3 months was associated with a significant reduction in MDA levels and a significant increase in serum TAS levels. Although considerable improvement was noted in these parameters, still there were significant differences from the control values. Our finding of enhanced oxidative stress and reduced TAS at baseline provides evidence for the presence of oxidative stress in newly diagnosed patients with T2DM. Furthermore, the decrease in levels of MDA, with a concomitant increase in TAS after three months of therapy seen in our study, may indicated a good response to therapy with either metformin alone or in combination with vitamin $\mathrm{E}$ and $\mathrm{C}$. These findings provided evidence for the presence of oxidative stress and inflammation in patients with T2DM. ${ }^{24}$ The findings of this study were in consistent with several studies which had been done to evaluate oxidative stress, antioxidant status, and markers of inflammation in diabetic patients. ${ }^{1,2}$ Increasing evidence in both experimental and clinical studies suggests that both oxidative stress and inflammation played a major role in the pathogenesis of both types of diabetes mellitus. $^{3}$

The present work revealed that there was a significant decrease in serum MDA levels in newly-diagnosed T2DM 
patients after a 3-month therapy with metformin monotherapy. These results were in consistent with other studies. ${ }^{29,30}$ Conversely, Skrha et al.(2007) observed that 3-month therapy with metformin was accompanied by significantly increased plasma MDA levels in T2DM patients. They concluded that initiation of metformin treatment in such patients was associated with activation of oxidative stress. ${ }^{31}$ Where as Gupta et al.(2010) observed no change in MDA levels at the end of the 12 weeks treatment with metformin, in newly diagnosed T2DM patients. ${ }^{32}$

The present study also revealed a significant increase $(P<0.01)$ in TAS of newly-diagnosed T2DM patients after a 3 -month metformin therapy, which is consistent with those reported by Tessier et al.(1999). ${ }^{33}$ Regarding the group of metformin with vitamins $\mathrm{C}$ and $\mathrm{E}$ combination, this study showed that they reported a significant reduction in serum levels of MDA and CRP with a significant increase in TAS after 3 months of treatment in comparison with their levels before therapy which is consistent with those reported by Ceriello and Testa (2009) whom observed that vitamins $\mathrm{C}$ and $\mathrm{E}$ supplements might help counteract the heightened oxidative and inflammatory conditions observed in diabetes and thus serve as an adjunctive therapeutic agent for the prevention and even treatment of the cardiovascular complications associated with diabetes. ${ }^{12}$ Moreover, these two vitamins which are important antioxidants in humans have the ability of scavenging oxygen-derived free radicals, improved hyperlipidemia and decreased blood pressure. ${ }^{34,35}$

A number of epidemiological studies demonstrated an inverse association between vitamin $\mathrm{E}$ and markers of oxidation, inflammation, and T2DM incidence. . $^{38,39,40}$

Our study showed a highly significant negative correlation between serum MDA levels and TAS in patients with diabetes, which suggests increased utilization by reactive oxygen species as an important contributing factor to the lower concentrations of antioxidants in patients with diabetes.

The result of the present study also showed that the serum CRP level in newly diagnosed patients with T2DM was significantly higher than those of healthy controls and that the administration of metformin alone or in combination with vitamins $\mathrm{C}$ and $\mathrm{E}$ to such patients for a duration of 3 months was associated with a significant decrease in serum CRP levels. Many studies showed that CRP is a reliable marker of cardiovascular disease (CVD) and had thus been incorporated into CVD risk prediction protocols especially in patients with diabetes mellitus. ${ }^{25,26}$ Regarding metformin therapy, the findings of this study were in consistent with the results of previous studies, as Akbar (2003) found that serum CRP level in well-controlled T2DM patients with metabolic syndrome was significantly lower in patients using metformin compared with those using glibenclamide. ${ }^{27}$ Other study that were in consistent with the finding of our results were those reported by Kahn, et al.(2010). ${ }^{28}$ Where as Tousoulis et al.(2007) reported that treatment with $2000 \mathrm{mg} /$ day for 4 weeks had no effect on levels of CRP, IL- 6 , TNF- $\alpha$, or soluble vascular cell adhesion molecule1 in 13 T2DM patients. ${ }^{36}$

Overall, Paolisso et al.(1995) reported that it may remain unclear whether vitamin $\mathrm{C}$ intake has an effect on factors related to T2DM. Although the epidemiologic evidence suggests that 
vitamin $\mathrm{C}$, whether as a supplement or as part of a diet rich in fruits and vegetables, beneficially affects inflammatory markers and disease risk, the results of intervention trials in T2DM are conflicting. ${ }^{37}$

In the present study, it has been found that diabetic patients with higher serum CRP concentrations, which is an indicative of greater inflammatory response, also had a higher serum MDA levels and lower TAS. This can be observed in Table 7, in which serum CRP level was positively correlated with lipid peroxidation products as MDA and negatively correlated with TAS. This finding was in consistence with Ahmed et al.(2003) whom they found that CRP level was positively associated with markers of oxidative stress (8isoprostanes). ${ }^{41}$

Interestingly, we did not observe any statistically significant differences in serum MDA levels between patients who received metformin alone, and patients who received metformin with the vitamins in combination. In fact, the serum level of TAS was found to be significantly higher, while serum level of CRP was significantly lower in patients who received metformin with the vitamins combination in comparison with metformin alone. This high level of TAS in the serum of diabetic patients who received the antioxidant vitamins together with metformin was most likely caused by the supplementary antioxidant effects of vitamins $\mathrm{C}$ and $\mathrm{E}$ that potentiates the effects of metformin on oxidative stress and inflammation. These finding were found consistent with those reported by other workers. $^{42,43,44}$

In conclusion, the present study found that three months oral consumption of combined antioxidant vitamins $\mathrm{C}$ and $\mathrm{E}$ with metformin in patients with T2DM showed significantly beneficial effect on oxidative stress and inflammatory response as compared with metformin alone. The findings of this study necessitate investigating the overall clinical impact of antioxidant treatment of type 2 diabetes mellitus to maintain good glycemic control.

\section{References}

1. Meigs JB, Larson MG, Fox CS, et al. Association of oxidative stress, insulin resistance and diabetes. Risk phenotype: The Framingham offspring study. Diabetes Care study. Diabetes Care 2007;30:2529-2535.

2. Badawi A, Klip A, Haddad P, et al. T2DM and inflammation: prospects for biomarkers of risk and nutritional intervention. Diabetes Metab Syndr Obes 2010;3:173-186.

3. Jin $\mathrm{Y}$, Han $\mathrm{G}, \mathrm{Hu} \mathrm{Y}$, et al. Tea consumption and risk of type 2 diabetes: A meta-analysis of cohort studies. J Gen Intern Med 2009;24:557-562.

4. Medina-Navarro R, Duran-Reyes G, Diaz-Flores $\mathrm{M}$, et al. Protein antioxidant response to the stress and the relationship between molecular structure and antioxidant function. PLOS One 2010;5(1):e8971.

5. Fridlyand LE, Philipson LH. Reactive species and early manifestation of insulin resistance in type 2 diabetes. Diabetes Obes Metab2006;8(2):136-145.

6. Takayanagi R, Inoguchi T, Ohnaka $\mathrm{K}$. Clinical and experimental evidence for oxidative stress as an exacerbating factor of diabetes mellitus. J Clin Biochem Nutr 2011;48(1):72-77.

7. Mugabo Y, Li L, Renier G. The connection between C-reactive protein (CRP) and diabetic 
vasculopathy. Focus on preclinical findings. Curr Diabetes Rev 2010;6(1):27-34.

8. Cox AJ, Agarwal SM, Herrington $\mathrm{D}$, et al. C-reactive protein concentration predicts mortality in type 2 diabetes: the Diabetes Heart Study. Diabet Med 2012;29(6):767770.

9. Abbatecola AM, Maggi S, Paolisso G. New approaches to treating type 2 diabetes mellitus in the elderly: role of incretin therapies. Drugs Aging 2008;25(11):913-925

10. Knowler WC, Barrett-Connor E, Fowler SE, et al. Reduction in the incidence of type 2 diabetes with lifestyle intervention or metformin. N Engl J Med 2002;346(6):393-403.

11. Levy J, Cobas RA, Gomes MB. Assessment of efficacy and tolerability of once-daily extended release metformin in patients with type 2 diabetes mellitus. Diabetol Metab Syndr 2010;2:16.

12. Ceriello A, Testa R. Antioxidant anti-inflammatory treatment in type 2 diabetes. Diabetes Care 2009;32(Suppl 2):S232-S236.

13. Lu Q, Bjorkhem I, Wretlind B, et al. Effect of ascorbic acid on microcirculation in patients with type II diabetes: a randomized placebocontrolled cross-over study.Clin Sci 2005;108(6):507-513.

14. Wannamethee SG, Lowe GD, Rumley A, et al. Associations of vitamin $\mathrm{C}$ status, fruit and vegetable intakes, and markers of inflammation and hemostasis. Am J Clin Nutr 2006;83(3):567-574.

15. Upritchard JE, Sutherl WHF, Mann JI. Effect of supplementation, vitamin $\mathrm{E}$ and vitamin $\mathrm{C}$ on $\mathrm{LDL}$ oxidation and products of inflammatory activity in type 2 diabetes. Diabetes Care 2008;23:733-738.

16. Ward NC, Wu JH, Clarke MW, et al. The effect of vitamin $\mathrm{E}$ on blood pressure in individuals with type 2 diabetes: a randomized, doubleblind, placebo-controlled trial. J Hypertens 2007;25(1):227-234.

17. Wu JH, Ward NC, Indrawan AP, et al. Effects of alpha-tocopherol and mixed tocopherol supplementation on markers of oxidative stress and inflammation in type 2 diabetes. Clin Chem 2007;53(3):511-519.

18. Kaviarasan K, Arjunan MM, Pugalendi KV. Lipid profile,oxidantantioxidant status and glycoprotein components in hyperlipidemic patients with/without diabetes. Clin Chim Acta 2005;362 (1-2):49-56.

19. ADA (American Diabetes Association). Standards of Medical Care in Diabetes: Position Statement. Diabet Care 2010;33:S11-61.

20. WHO (World Health Organization). Definition and Diagnosis of Diabetes Mellitus and Intermediate Hyperglycemia: Report of a WHO/ IDF consultation. Geneva: WHO Document Production Services 2006;1-41.

21. Buege JA, Aust SD. The thiobarbituric acid assay. Methods in Enzymology 1978;52: 306-310.

22. Miller NJ, Rice-Evans C, Davies MJ, et al. A novel method for measuring antioxidant capacity and its application to monitoring the antioxidant status in premature neonates. Clin Sci 1993;84:407-412.

23. Kirkwood BR. Essentials of Medical Statistics.1st ed. Oxford: Blackwell Scientific Publications1988:43p.

24. Kathryn E, Gökhan S. Inflammation, stress, and diabetes. J Clin Invest 2005;115(5):1111-1119. 
25. Kaptoge S, Di Angelantonio E, Pennells L, et al. C-reactive protein, fibrinogen, and cardiovascular disease prediction. $\mathrm{N}$ Engl J Med 2012;367(14):13101320.

26. Torres J1, Ridker PM. Clinical use of high sensitivity C-reactive protein for the prediction of adverse cardiovascular events. Curr Opin Cardiol 2003.18:471-478.

27. Akbar DH. Effect of metformin and sulfonylurea on C-reactive protein level in well-controlled type 2 diabetes with metabolic syndrome. Endocrine 2003;20:215-218.

28. Kahn SE, Haffner SM,Viberti G, et al. Rosiglitazone decreases Creactive protein to a greater extent relative to glyburide and metformin over 4 years despite greater weight gain: Observation from a diabetes outcome progression trial (ADOPT). Diabet Care 2010;33:177-183.

29. Pavlović D, Kocić R, Kocić G, et al. Effect of four-week metformin treatment on plasma and erythrocyte antioxidative defense enzymes in newly diagnosed obese patients with type 2 diabetes. Diabetes Obes Metab 2000;2:251-256.

30. Alaa A, Imad A. Comparative Effects of Glibenclamide and Metformin on C-Reactive Protein and Oxidant/Antioxidant Status in Patients with Type II Diabetes Mellitus. Sultan Qaboos Univ Med J 2012;12(1): 55-61.

31. Skrha J, Prázný M, Hilgertová J, et al. Oxidative stress and endothelium influenced by metformin in type 2 diabetes mellitus. Eur J Clin Pharmacol 2007;63(12):1107-1114.

32. Gupta RK, Rehan HS, Rohatagi A, et $a l$. The effect of glipizide, metformin and rosiglitazone on non-traditional cardiovascular risk factors in newly diagnosed patients with type 2 diabetes mellitus. Int J Diabetes Dev Ctries 2010;30:123-128.

33. Tessier D, Maheux P, Khalil A, et al. Effects of gliclazide versus metformin on the clinical profile and lipid peroxidation markers in type 2 diabetes. Metabolism1999; 48:897903.

34. Vega-Lopez S, Devaraj S, Jialal I. Oxidative stress and antioxidant supplementation in the management of diabetic cardiovascular disease. $\mathbf{J}$ Investig Med 2004;52(1):24-32.

35. Chen H, Karne RJ, Hall G, et al. High-dose oral vitamin C partially replenishes vitamin $\mathrm{C}$ levels in patients with type 2 diabetes and low vitamin $\mathrm{C}$ levels but does not improve endothelial dysfunction or insulin resistance. Am J Physiol Heart Circ Physiol 2006;290(1):H137-H145.

36. Tousoulis D, Antoniades C, Vasiliadou C, et al. Effects of atorvastatin and vitamin $\mathrm{C}$ on forearm hyperaemic blood flow, asymmentrical dimethylarginine levels and the inflammatory process in patients with type 2 diabetes mellitus. Heart 2007;93(2):244-246.

37. Paolisso G, Balbi V, Volpe C, et al. Metabolic benefits deriving from chronic vitamin $\mathrm{C}$ supplementation in aged non-insulin dependent diabetics. J Am Coll Nutr 1995;14(4):387-392.

38. Salonen JT, Nyyssonen K, Tuomainen TP, et al. Increased risk of non-insulin dependent diabetes mellitus at low plasma vitamin $\mathrm{E}$ concentrations: a four year follow up study in men. BMJ 1995;311(7013):1124-1127.

39. Garcia-Bailo B, El-Sohemy A, Haddad PS, et al. Vitamins D, C, 
and $\mathrm{E}$ in the prevention of type 2 diabetes mellitus: modulation of inflammation and oxidative stress. Biologics 2011;5:7-19.

40. Ristow M, Zarse K, Oberbach A, et al. Antioxidants prevent healthpromoting effects of physical exercise in humans. Proc Natl Acad Sci USA 2009;106(21):8665-8670.

41. Ahmed A, Jennifer C. Relationship between Oxidative Stress and Inflammatory Cytokines in Diabetic Nephropathy. Cardiovascular Therapeutics 2012. 30(1):49-59.
42. Maritim AC, Sanders RA, Watkins JB. Diabetes, oxidative stress, and antioxidants: a review. J Biochem Mol Toxicol 2003;17(1):24-38.

43. Rahimi R, Nikfar S, Larijani B, et al. A review on the role of antioxidants in the management of diabetes and its complications. Biomed Pharmacother 2005;59(7):365-373.

44. Golbidi S, Ebadi SA, Laher I. Antioxidants in the treatment of diabetes. Curr Diabetes Rev 2011;7(2):106-125. 